\title{
Transformation method and wave control
}

\author{
Zheng Chang · Jin Hu • Geng-Kai Hu
}

Received: 13 September 2010 / Revised: 11 October 2010 / Accepted: 29 October 2010 / Published online: 22 December 2010

(C) The Author(s) 2010. This article is published with open access at Springerlink.com

\begin{abstract}
Transformation method provides an efficient way to control wave propagation by materials. The transformed relations for field and material during a transformation are essential to fulfill this method. We propose a systematic method to derive the transformed relations for a general physic process, the constraint conditions are obtained by considering geometrical and physical constraint during a mapping. The proposed method is applied to Navier's equation for elastodynamics, Helmholtz's equation for acoustic wave and Maxwell's equation for electromagnetic wave, the corresponding transformed relations are derived, which can be used in the framework of transformation method for wave control. We show that contrary to electromagnetic wave, the transformed relations are not uniquely determined for elastic wave and acoustic wave, so we have a freedom to choose them differently. Using the obtained transformed relations, we also provide some examples for device design, a concentrator for elastic wave, devices for illusion acoustic and illusion optics are conceived and validated by numerical simulations.
\end{abstract}

Keywords Wave control Elastic wave - Acoustic wave . Electromagnetic wave $\cdot$ Local affine transformation

The project was supported by the National Natural Science Foundation of China (10832002), and the National Basic Research Program of China (2006CB601204).

Z. Chang · G.-K. Hu (凶)

School of Aerospace Engineering, Beijing Institute of Technology, 100081 Beijing, China

e-mail: hugeng@bit.edu.cn

J. Hu

School of Information and Electronics,

Beijing Institute of Technology, 100081 Beijing, China

\section{Introduction}

Controlling wave propagation by spatial arrangement of materials is a topic of active researches from different horizons, e.g., from wave mechanics for heterogeneous materials, to inverse problem and engineering applications for stealth technology and noise isolations. Recently emerge of metamaterial, a composite with unusual effective property by tailoring its microstructure, provides an unprecedented possibility for material selection. Materials with negative effective permittivity or permeability for electromagnetic (EM) waves $[1,2]$, or with negative effective density or modulus for acoustic waves [3-6] can be readily fabricated. Particularly, materials with double negative material parameters, called left-handed materials can bend waves with negative refractive index [1]. The development of metamaterials paves the way for controlling wave with materials, we can have any material property at hand to manipulate waves. To guide waves in a prescribed manner, we need to know how to place spatially different materials, this is a typical inverse problem [7] which will involve a heavy mathematical technique due to non-unique solution. To circumvent this difficulty, Greenleaf et al. [8], Pendry et al. [9] and Leonhardt [10] proposed a transformation method which can construct directly one solution of the above inverse problem for electromagnetic wave. The basic principle is based on the form-invariance of Maxwell's equations under a general spatial mapping, i.e., Minkowski's expression in a curvilinear system, so a topological effect can be mimicked by materials. For example, from a virtual space of vacuum, we choose a region with a fixed boundary, and enlarge from a point inside of this region to form a cavity, the region is distorted by this action. The effect of this spatial distortion can be simulated by material spatial distribution in a physical space due to 
a

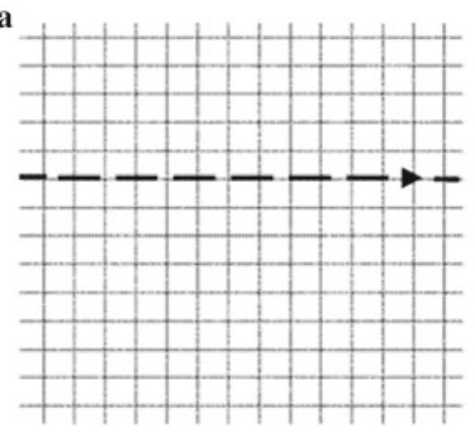

b

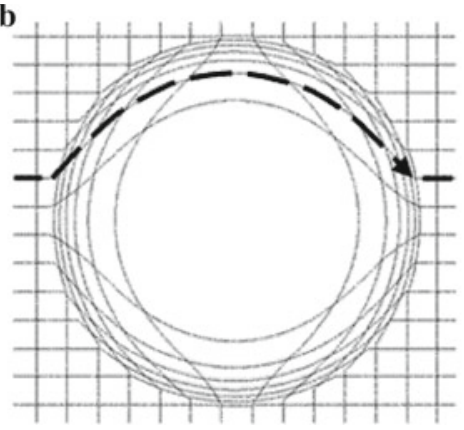

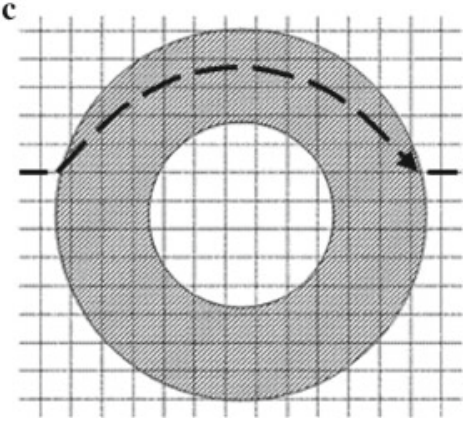

Fig. 1 Principle of transformation method. a Virtual space, $\mathbf{b}$ distorted space, $\mathbf{c}$ physical space

form-invariance of Maxwell's equation [11]. So in the physical space (Fig. 1c), an observer outside the distorted region perceives the same EM wave characteristic as in the virtual space (Fig.1a), anything inside of the cavity cannot be detected by the outside observer, therefore forming an invisible cloak $[9,12]$.

We can go a step further by folding a part of the virtual space, a complementary medium is then constructed which can conceal its pair-object [13] in context of EM wave, or eventually we can make a real spoon to look apparently as a cup instead, constructing an illusion cloak [14]. Many interesting devices can be imagined, however the basic principle lies in equivalency between geometry and media for EM wave [15]. The interplay between gravity and geometry established by general relativity also reveals that gravitational effect on photon in cosmology can be simulated by a media in a laboratory [16]. The transformation method is not limited only to EM wave, it is shown that Helmholtz's equation for acoustic wave is also form-invariant under a general mapping [17], so acoustic wave also perceives a geometry as a media, many known results for EM wave can be directly transported to acoustic wave $[18,19]$. The same conclusions can also be made for heat conduction [20] and matter wave [21].

To control wave propagation by transformation method, two actions are necessary: first a topological transformation defining the function of a device, or the pattern that wave will follow; second transformed relations point by point for field and material from a virtual (initial) space to a physical (transformed) space. Unlike EM wave which has a clear and definite transformed relation, the transformed relations for acoustic wave, depending on the transformed medium, can have many possibilities [18,22-25]. As far as for elastic wave, the progress is much limited, transformed relation for elastic wave cannot be derived from the previous method due to lack of form-invariance for Navier's equation. However, some special cases can still be examined: for example in quasistatic limit, an elastic cloak with a homogeneous metamaterial [26] or cylindrical cloak for bending wave in a thin plate [27]. Recently, we proposed a general method to derive the transformed relation for any governing differential equation without necessity to be form-invariant in any arbitrary curvilinear coordinate system [25], the transformed relation for elastic wave can also be obtained [28]. In this paper, we will summarize the progress of the works and present them in a unified way for different waves. The paper is arranged as follows: the deformation view of the transformation method and the constraint conditions for transformed relation will be explained in Sect. 2; the general concept will be separately applied to elastic wave, acoustic wave and electromagnetic wave. The results with new applications will subsequently be given in Sects. 3, 4 and 5, and followed by some conclusions.

\section{Transformation and related physical process}

\subsection{Transformation method}

Consider a physical process described on an initial space (or virtual space) $\Omega$, the field $\boldsymbol{u}$ and material $\boldsymbol{C}$ are related together at a point $\boldsymbol{x}$ and time $t$ by a differential equation $F$ as

$F(x, t, u(x, t), C(x))=0, \quad x \in \Omega$.

The operator $F$ will be specified for elastic wave, acoustic wave or EM wave in the subsequent sections. Here, we prefer keeping our discussion as general as possible. The operator $F$ monitors spatially and temporally the dance of the pair $\boldsymbol{u}$ and $\boldsymbol{C}$, characterizing the relation between the field and material. The idea of transformation method is to perform a mapping, which maps the space $\Omega$ to $\Omega^{\prime}$ (transformed space or physical space) by $\boldsymbol{x}^{\prime}=\boldsymbol{x}^{\prime}(\boldsymbol{x})$

$$
M:\{x: x \in \Omega\} \mapsto\left\{x^{\prime}: x^{\prime} \in \Omega^{\prime}\right\},
$$


and the attached field $\boldsymbol{u}$ and material $\boldsymbol{C}$ are also mapped to $\Omega^{\prime}$, symbolically written as

$\boldsymbol{u}^{\prime}\left(\boldsymbol{x}^{\prime}, t\right)=\boldsymbol{T}_{\mathrm{u}}[\boldsymbol{u}(\boldsymbol{x}, t)], \quad \boldsymbol{C}^{\prime}\left(\boldsymbol{x}^{\prime}, t\right)=\boldsymbol{T}_{\mathrm{C}}[\boldsymbol{C}(\boldsymbol{x}, t)]$.

$\boldsymbol{T}_{\mathrm{u}}, \boldsymbol{T}_{\mathrm{C}}$ are the transformation operators for the field and material, respectively. Equation (3) is called transformed relation, which is crucial for controlling waves by a mapping. For example, we can choose carefully the mapping $M$ such that the transformed field $\boldsymbol{u}^{\prime}$ in the physical space follows a designed way, the last term of Eq. (3) tells how to realize this function by the material distribution. Usually the mapping characterizes the function to be realized, the transformation relation tells how to realize the functional device by material and the field distribution in the device. To establish the transformation relation, according to transformation method, we have to express the governing equation in a general curvilinear system. Taking Maxwell's equation as an example, in a Cartesian system established in the virtual space, it can be expressed as

$\nabla \times \boldsymbol{E}=-\boldsymbol{\mu} \frac{\partial \boldsymbol{H}}{\partial t}, \quad \nabla \times \boldsymbol{H}=+\boldsymbol{\varepsilon} \frac{\partial \boldsymbol{E}}{\partial t}$.

For a general mapping $\boldsymbol{x}^{\prime}=\boldsymbol{x}^{\prime}(\boldsymbol{x})$, characterizing by a transformation tensor $\boldsymbol{A}=\partial \boldsymbol{x}^{\prime} / \partial \boldsymbol{x}$, which maps the initial space to the physical (or transformed) space, Maxwell's equation written in a curvilinear system is

$$
\nabla \times \boldsymbol{E}^{\prime}=-\boldsymbol{\mu}^{\prime} \frac{\partial \boldsymbol{H}^{\prime}}{\partial t}, \quad \nabla \times \boldsymbol{H}^{\prime}=+\boldsymbol{\varepsilon}^{\prime} \frac{\partial \boldsymbol{E}^{\prime}}{\partial t} .
$$

Due to the invariance of Maxwell's equation, the geometrical effect induced by the mapping can be mimicked by spatial material distribution. Comparing Eq. (4) with (5), the transformed relation for material parameters in the physical space can be related to those in the initial space and to the transformation tensor $\boldsymbol{A}$ by

$$
\boldsymbol{\varepsilon}^{\prime}\left(\boldsymbol{x}^{\prime}\right)=\frac{\boldsymbol{A} \boldsymbol{\varepsilon}(\boldsymbol{x}) \boldsymbol{A}^{\mathrm{T}}}{\operatorname{det} \boldsymbol{A}}, \quad \boldsymbol{\mu}^{\prime}\left(\boldsymbol{x}^{\prime}\right)=\frac{\boldsymbol{A} \boldsymbol{\mu}(\boldsymbol{x}) \boldsymbol{A}^{\mathrm{T}}}{\operatorname{det} \boldsymbol{A}}
$$

For acoustic wave governed by Helmholtz's equation, the same technique can also be applied thanks to the invariance of Helmholtz's equation under a general transformation. However for elastic wave, it is shown that Navier's equation is not form-invariant [22] in an arbitrary curvilinear coordinate system, so the following question arises: are there transformed relations for controlling elastic wave? To answer this question, we will reconsider the transformation itself and examine how the field and material are transported to a new space (physical space).

\subsection{Deformation view of transformation method}

The mapping given by Eq. (2) can be considered as a deformation field from the initial space $\Omega$ to the transformed space $\Omega^{\prime}$, i.e. a point and its infinitesimal region in $\Omega$ are affinely transformed into a new point and another infinitesimal region in $\Omega^{\prime}$. According to continuum mechanics [29], the deformation gradient $\boldsymbol{A}$ induced by a mapping can be decomposed by a rotation (orthogonal) tensor $\boldsymbol{R}$ and a pure stretch tensor $\boldsymbol{V}$ as $\boldsymbol{A}=\boldsymbol{V} \boldsymbol{R}$. In order to proceed, we define at each point $\mathbf{e}_{i}^{\prime}$ and $\lambda_{i}$, respectively, the eigenvectors and eigenvalues of $\boldsymbol{V}$, i.e., $\boldsymbol{V}=\lambda_{1} \mathbf{e}_{1}^{\prime} \mathbf{e}_{1}^{\prime}+\lambda_{2} \mathbf{e}_{2}^{\prime} \mathbf{e}_{2}^{\prime}+\lambda_{3} \mathbf{e}_{3}^{\prime} \mathbf{e}_{3}^{\prime}$, $\mathbf{e}_{i}^{\prime}$ establishes a local Cartesian system $\mathbf{e}^{\prime}$ in the transformed space. By this decomposition, the effects on a spatial element by the mapping are twofold: the element is firstly rotated to the system $\mathbf{e}^{\prime}$, then it is stretched along $\mathbf{e}_{i}^{\prime}$ by a factor $\lambda_{i}$ in the local Cartesian system. Now we will examine how the attached physical field and material with the element are transformed during the mapping. We can imagine the following process: the physical field $\boldsymbol{u}$ and material $\boldsymbol{C}$ in the initial space are rigidly rotated with the element to the local system $\mathbf{e}^{\prime}$ of the physical space, in the new local Cartesian system $\mathbf{e}^{\prime}$ the components of the field $\boldsymbol{u}$ and material $\boldsymbol{C}$ will not change; then in the local system they will be rescaled along $\mathbf{e}_{i}^{\prime}$, as shown in Fig. 2. So the transformed field $\boldsymbol{u}^{\prime}$ and material $\boldsymbol{C}^{\prime}$ can be written symbolically in the local system $\mathbf{e}^{\prime}$ as

$\boldsymbol{u}^{\prime}=\boldsymbol{V}_{\mathrm{u}}[\boldsymbol{u}], \quad \boldsymbol{C}^{\prime}=\boldsymbol{V}_{\mathrm{C}}[\boldsymbol{C}]$.

$\boldsymbol{V}_{\mathrm{u}}, \boldsymbol{V}_{\mathrm{C}}$ are rescaling operators for the field and material, respectively, which remain to be determined. The rescaling operator can be denoted by a second order tensor $\boldsymbol{a}$, and its operation on a physical quantity representing by a tensor should follows a tensor transformation rule. In the specially established local Cartesian system, the stretch tensor has a diagonal form $\boldsymbol{a}=\operatorname{diag}\left(\alpha_{1}, \alpha_{2}, \alpha_{3}\right)$, its operation on a vector $d_{i}$, a second order tensor $d_{i j}$ and a forth order tensor $d_{i j k l}$ can be written, respectively, as $d_{i}^{\prime}=\alpha_{I} d_{i}, d_{i j}^{\prime}=$ $\alpha_{I} \alpha_{J} d_{i j}, d_{i j k l}^{\prime}=\alpha_{I} \alpha_{J} \alpha_{K} \alpha_{L} d_{i j k l}$, where $\delta_{i j}$ is Kronecker delta, $\alpha_{i}$ is called scaling factor in the following. The capital letter in index means the same value as its lower case but without summation.

Consider a line element $\mathrm{d} x_{i}$ in a Cartesian system (e) of the initial space, it is deformed to the physical space by the mapping. In the local Cartesian system $\mathbf{e}^{\prime}$, we have $\mathrm{d} x_{i}^{\prime}=\lambda_{i} \mathrm{~d} x_{i}$, leading to the following differential relation between the two spaces

$$
\frac{\partial}{\partial x_{i}^{\prime}}=\frac{\partial}{\lambda_{i} \partial x_{i}} \text {. }
$$

Equations (7) and (8) provide in fact geometrical constraint for the transformation relation, however they have to be subjected to physical constraints as well. Firstly the transformed field and material in the physical space should reconstruct 
Fig. 2 Sketch of deformation for an element and the attached physical quantity. a Initial space, $\mathbf{b}$ transformed space a

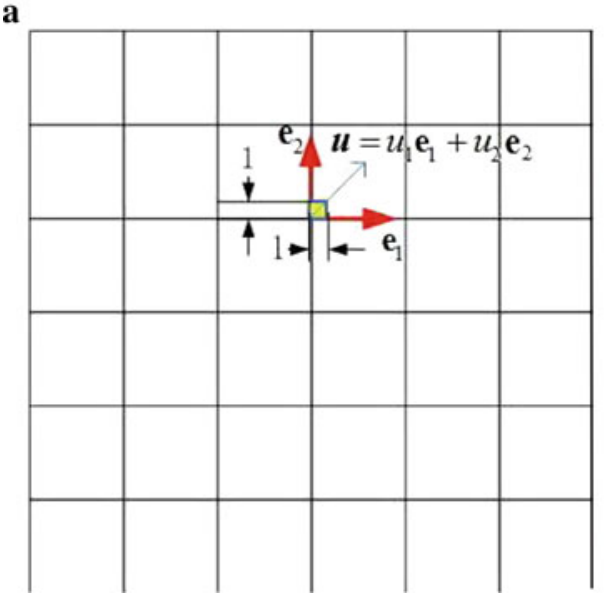

b

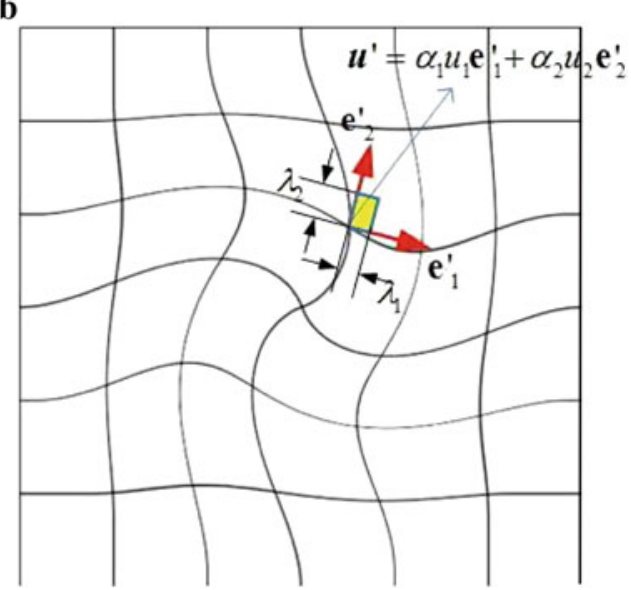

the same physical process as that in the initial space, namely in the local Cartesian system $\mathbf{e}^{\prime}$

$F\left(\boldsymbol{x}^{\prime}, t, \boldsymbol{u}^{\prime}(\boldsymbol{x}, t), \boldsymbol{C}^{\prime}\left(\boldsymbol{x}^{\prime}\right)\right)=0, \quad \boldsymbol{x}^{\prime} \in \Omega^{\prime}$.

Equation (9) means that in the two Cartesian systems, the governing equation of the same physical process should be the same. This can be always done in the local sense for the considered local affine mapping round a point where Christoffel symbol vanishes at this point. In addition, we assume during the mapping, each type of energy is conserved and there is not energy conversion. If $\boldsymbol{w}(\boldsymbol{u}, \boldsymbol{C})$ and $w^{\prime}\left(\boldsymbol{u}^{\prime}, \boldsymbol{C}^{\prime}\right)$ denote, respectively, any type of energy densities in the initial and transformed spaces, the volume of an element $\mathrm{d} v$ becomes $\lambda_{1} \lambda_{2} \lambda_{3} \mathrm{~d} v$ during the mapping, so the energy conservation of each point leads to the following physical constraint condition

$w(\boldsymbol{u}, \boldsymbol{C})=w^{\prime}\left(\boldsymbol{u}^{\prime}, \boldsymbol{C}^{\prime}\right) \lambda_{1} \lambda_{2} \lambda_{3}$.

The constraint conditions given by Eqs. (7)-(10) will allow us finally to determine the transformed relations for the field and material if a physical process is prescribed [30]. In the following, the above general method will be applied to elastic, acoustic and electromagnetic waves, respectively.

\section{Transformation elastodynamics}

\subsection{Transformed relation for Navier's equation}

Now we specify the governing equation $F$ in Sect. 2 for an elastodynamic problem, the physical field variables are respectively stress $\boldsymbol{\sigma}$ and displacement $\boldsymbol{u}$, the material parameters are modulus tensor $\boldsymbol{C}$ and density $\rho$. They are related by the following Navier's equation and constitutive equation

$\nabla \cdot \boldsymbol{\sigma}=\rho \frac{\partial^{2} \boldsymbol{u}}{\partial t^{2}}, \quad \boldsymbol{\sigma}=\boldsymbol{C}: \nabla \boldsymbol{u}$, or written in a Cartesian system e in an index form as

$\frac{\partial \sigma_{i j}}{\partial x_{i}}=\rho \frac{\partial^{2} u_{j}}{\partial t^{2}}, \quad \sigma_{i j}=C_{i j k l} \frac{\partial u_{k}}{\partial x_{l}}$.

Now the field and material are transformed from an initial space to a physical space with help of a mapping $\boldsymbol{A}$. The scaling tensors for different quantities are different but they are assumed to have a diagonal form written in the local Cartesian system e. So the transformed field and material are related to the field and material in the initial space as well as the scaling factors as

$\sigma_{i j}^{\prime}=\alpha_{I} \alpha_{J} \sigma_{i j}, \quad u_{i}^{\prime}=\beta_{I} u_{i}$,

$C_{i j k l}^{\prime}=\chi_{I} \chi_{J} \chi_{K} \chi_{L} C_{i j k l}, \quad \rho_{i j}^{\prime}=\varphi_{I} \delta_{i j} \rho$,

where $\alpha_{i}, \beta_{i}, \chi_{i}, \varphi_{i}(i=I, J, K, L)$ are scaling factors for the stress, displacement, stiffness and density, respectively. The density is assumed to be a second order tensor [31]. As discussed in Sect. 2.2, the transformed field and material in the local Cartesian system $\mathbf{e}^{\prime}$ should satisfy the Navier's equation, i.e.,

$\frac{\partial \sigma_{i j}^{\prime}}{\partial x_{i}^{\prime}}=\rho_{i j}^{\prime} \frac{\partial^{2} u_{i}^{\prime}}{\partial t^{2}}, \quad \sigma_{i j}^{\prime}=C_{i j k l}^{\prime} \frac{\partial u_{k}^{\prime}}{\partial x_{l}^{\prime}}$.

We put Eq. (13) into Eq. (14), and with help of Eq. (8), Eq. (14) can be written with the variable, field and material in the local system $\mathbf{e}$ of in the initial space as,

$\frac{\alpha_{I} \alpha_{J}}{\lambda_{I}} \frac{\partial \sigma_{i j}}{\partial x_{i}}=\varphi_{J} \beta_{J} \rho \frac{\partial^{2} u_{j}}{\partial t^{2}}$,
$\alpha_{I} \alpha_{J} \sigma_{i j}=\chi_{I} \chi_{J} \chi_{K} \chi_{L} \frac{\beta_{K}}{\lambda_{L}} C_{i j k l} \frac{\partial u_{k}}{\partial x_{l}}$.

Comparing directly Eq. (15) with Eq. (12), this leads to the following relations between the scaling factors and the stretch tensor at each point,

$\frac{\alpha_{i} \alpha_{j}}{\lambda_{I}}=\varphi_{j} \beta_{J}, \quad \alpha_{i} \alpha_{j}=\chi_{i} \chi_{j} \chi_{K} \chi_{L} \frac{\beta_{k}}{\lambda_{l}}$. 
The kinetic and strain energy conservations at each point during the mapping imply that

$$
\begin{aligned}
& \rho \frac{\partial u_{i}}{\partial t} \frac{\partial u_{i}}{\partial t}=\lambda_{1} \lambda_{2} \lambda_{3} \rho_{i j}^{\prime} \frac{\partial u_{i}^{\prime}}{\partial t} \frac{\partial u_{j}^{\prime}}{\partial t}, \\
& \sigma_{i j}\left(\frac{\partial u_{i}}{\partial x_{j}}+\frac{\partial u_{j}}{\partial x_{i}}\right)=\lambda_{1} \lambda_{2} \lambda_{3} \sigma_{i j}^{\prime}\left(\frac{\partial u_{i}^{\prime}}{\partial x_{j}^{\prime}}+\frac{\partial u_{j}^{\prime}}{\partial x_{i}^{\prime}}\right) .
\end{aligned}
$$

With help of Eqs. (8) and (13), we can rewrite the right-hand terms of Eq. (17) with the variables of the initial space, leading to

$$
\begin{aligned}
& \rho \frac{\partial u_{i}}{\partial t} \frac{\partial u_{i}}{\partial t}=\lambda_{1} \lambda_{2} \lambda_{3} \varphi_{I} \delta_{i j} \rho \beta_{I} \beta_{J} \frac{\partial u_{i}}{\partial t} \frac{\partial u_{j}}{\partial t}, \\
& \sigma_{i j}\left(\frac{\partial u_{i}}{\partial x_{j}}+\frac{\partial u_{j}}{\partial x_{i}}\right)=\lambda_{1} \lambda_{2} \lambda_{3} \alpha_{I} \alpha_{J} \sigma_{i j}\left(\frac{\beta_{I}}{\lambda_{J}} \frac{\partial u_{i}}{\partial x_{j}}+\frac{\beta_{J}}{\lambda_{I}} \frac{\partial u_{j}}{\partial x_{i}}\right) .
\end{aligned}
$$

Finally the following constraint relations are derived from the energy conservation

$\lambda_{1} \lambda_{2} \lambda_{3} \varphi_{i} \beta_{I}^{2}=1$,

$\lambda_{1} \lambda_{2} \lambda_{3} \alpha_{i} \alpha_{j} \frac{\beta_{I}}{\lambda_{J}}=\lambda_{1} \lambda_{2} \lambda_{3} \alpha_{i} \alpha_{j} \frac{\beta_{J}}{\lambda_{I}}=1$.

From Eqs. (16), (19), we arrive at the following relations which allow us to establish the relation between the scaling factors and the stretch tensor

$\frac{\alpha_{j} \alpha_{J}}{\varphi_{J} \beta_{J}}=\lambda_{i}, \quad \frac{\alpha_{i} \alpha_{j}}{\chi_{I} \chi_{J} \chi_{K} \chi_{l} \beta_{k}}=\frac{1}{\lambda_{l}}, \quad \alpha_{i} \alpha_{j} \beta_{I}=\frac{\lambda_{j}}{\lambda_{1} \lambda_{2} \lambda_{3}}$.

In Eq. (20), $\alpha_{i}, \beta_{i}, \chi_{i}, \varphi_{i}$ are the scaling factors to be determined, however in a general case they can not be uniquely determined. If one transformed relation is specified for either field or material, e.g., $\boldsymbol{u}^{\prime}=\left(\boldsymbol{A}^{\mathrm{T}}\right)^{-1} \boldsymbol{u}$ or $\beta_{i}=1 / \lambda_{i}$ as in Ref. [22], the following relation between the scaling factors and stretch tensor for the rest field and material can be uniquely derived,

$\alpha_{i} \alpha_{j}=\frac{\lambda_{i} \lambda_{j}}{\operatorname{det} \boldsymbol{A}}, \quad \beta_{i}=\frac{1}{\lambda_{i}}$,

$\chi_{i} \chi_{j} \chi_{k} \chi_{l}=\frac{\lambda_{i} \lambda_{j} \lambda_{k} \lambda_{l}}{\operatorname{det} \boldsymbol{A}}, \quad \varphi_{i}=\frac{\lambda_{i}^{2}}{\operatorname{det} \boldsymbol{A}}$.

Finally, the transformed relations are given by

$\sigma_{i j}^{\prime}=\frac{\lambda_{I} \lambda_{J}}{\operatorname{det} A} \sigma_{i j}, \quad u_{i}^{\prime}=\frac{1}{\lambda_{I}} u_{i}$,

$C_{i j k l}^{\prime}=\frac{\lambda_{I} \lambda_{J} \lambda_{K} \lambda_{L}}{\operatorname{det} \boldsymbol{A}} C_{i j k l}, \quad \rho_{i j}^{\prime}=\frac{\lambda_{I}^{2}}{\operatorname{det} \boldsymbol{A}} \delta_{i j} \rho$.

Alternatively, if we specify the transformed relation for the displacement as $\boldsymbol{u}^{\prime}=(\operatorname{det} \boldsymbol{A})\left(\boldsymbol{A}^{\mathrm{T}}\right)^{-1} \boldsymbol{u}$ or $\beta_{i}=(\operatorname{det} \boldsymbol{A}) / \lambda_{i}$, the following transformed relations can also be derived,

$$
\begin{aligned}
& \sigma_{i j}^{\prime}=\frac{\lambda_{I} \lambda_{J}}{(\operatorname{det} \boldsymbol{A})^{2}} \sigma_{i j}, \quad u_{i}^{\prime}=\frac{\operatorname{det} \boldsymbol{A}}{\lambda_{I}} u_{i}, \\
& C_{i j k l}^{\prime}=\frac{\lambda_{I} \lambda_{J} \lambda_{K} \lambda_{L}}{(\operatorname{det} \boldsymbol{A})^{3}} C_{i j k l}, \quad \rho_{i j}^{\prime}=\frac{\lambda_{I}^{2}}{(\operatorname{det} \boldsymbol{A})^{3}} \delta_{i j} \rho .
\end{aligned}
$$

It is seen that the derived transformed relations for material parameters have the necessary symmetry, which means that they are realizable contrary to those reported in [32]. In the following section, we will present an example to design an elastic concentrator to illustrate how to control elastic wave by the transformed relation given by Eq. (22).

\subsection{Numerical example for an elastic concentrator}

In the following section, we will design a circular region which can concentrate the elastic wave without reflection. To this end, we choose a two dimensional virtual space with a homogeneous material, two concentric circles with radius $c$ and $b$ are chosen, the outer boundary is fixed and the inner boundary is compressed to a small circle $a$ in which elastic wave will be concentrated, as shown in Fig. 3a. This action in fact specifies the boundary condition for the mapping which realizes the concentrator. A linear transformation relation is chosen for the inner region $(r<a)$, expressed in a cylindrical coordinate as

$r^{\prime}=\frac{a}{b} r, \quad \theta^{\prime}=\theta$.

The mapping of the region $b<r<c$ should be specially constructed in order to make the concentrator without reflection or scattering. The key point is to guarantee the $C^{1}$ continuity of the transformation relation at the boundaries $r=$ $c, r=a$. To this end, a third-order polynomial is proposed for the radial transformation

$r^{\prime}=m r^{3}+n r^{2}+s r+t, \quad \theta^{\prime}=\theta$,

with the following boundary conditions

$\left.r^{\prime}\right|_{r=c}=c,\left.r^{\prime}\right|_{r=b}=a,\left.\frac{\partial r^{\prime}}{\partial r}\right|_{r=c}=1,\left.\frac{\partial r^{\prime}}{\partial r}\right|_{r=b}=\frac{a}{b}$.

Once the mapping is determined, the components of the stretch tensor are computed directly by

$\lambda_{r}=\frac{\mathrm{d} r^{\prime}}{\mathrm{d} r}, \quad \lambda_{\theta}=\frac{r^{\prime}}{r} \frac{\mathrm{d} \theta^{\prime}}{\mathrm{d} \theta}$.

So the material necessary to realize the concentrator is directly given by Eq. (22). In order to verify the designed elastic concentrator, we will perform a numerical experiment with the material spatial distribution determined above. An approximate point source with S-wave illuminates the concentrator, the computed domain is surrounded by PML to mimic the infinite space [33], as shown in Fig. 3a. In the computation, we choose $a=0.1 \mathrm{~m}, b=0.2 \mathrm{~m}$ and $c=0.4 \mathrm{~m}$, 
a

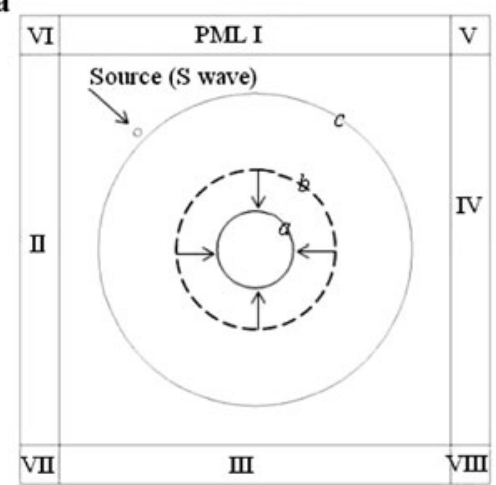

b

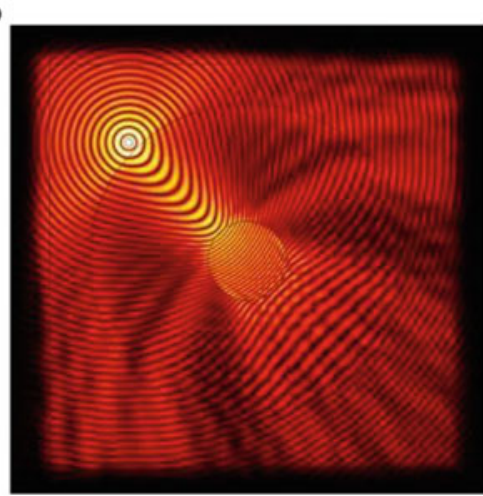

c

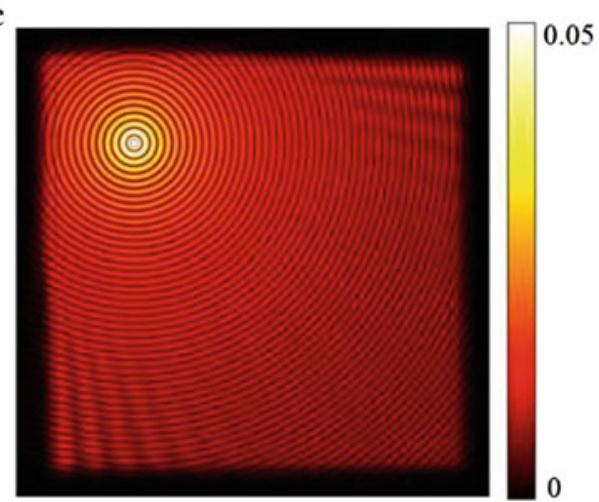

Fig. 3 Simulation of elastic concentrator. a The mapping and computed model, b total displacement field $\sqrt{u_{x}^{2}+u_{y}^{2}}$ with concentrator, $\mathbf{c}$ without concentrator

the background is set to be an isotropic material with normalized Lame constants $\lambda=2.3, \mu=1$ and normalized mass density $\rho=1$. A small circle used to simulate a point source of $\mathrm{S}$-wave is placed outside the concentrator. The static elastic wave equation $\nabla \cdot(\boldsymbol{C}: \nabla \boldsymbol{u})=-\rho \omega^{2} \boldsymbol{u}$ is solved with help of FEM software COMSOL Multiphysics. The computed results with and without concentrator are given by Fig. 3b and c, respectively. As shown in Fig. 3b, the elastic wave is indeed concentrated in the region $r<a$, as designed. The observed small scattering outside the designed concentrator is probably due to the numerical discretization in the case of strong material gradient.

\section{Transformation acoustics}

\subsection{Acoustic wave in classical fluid}

Governing equation of classical acoustic wave in inviscid fluid takes the following form

$\frac{\partial p}{\partial x_{i}}=\rho \frac{\partial^{2} u_{i}}{\partial t^{2}}, \quad p=\kappa \frac{\partial u_{i}}{\partial x_{i}}$,

where $p$ is pressure, $\boldsymbol{u}$ is displacement vector, $\rho$ is the mass density and $\kappa$ is the bulk modulus. Now we perform a mapping and transport $p, \boldsymbol{u}, \rho$ and $\kappa$ from an initial space to a transformed space, further the transformed material is assumed to be a classical fluid, i.e., the material with anisotropic mass density and isotropic stiffness [22]. Then at any point in the transformed space, the following transformed relations in the local Cartesian system $\mathbf{e}^{\prime}$ can be written as

$p^{\prime}=\alpha p, \quad u_{i}^{\prime}=\beta_{I} u_{i}, \quad \kappa^{\prime}=\chi \kappa, \quad \rho_{i j}^{\prime}=\varphi_{I} \delta_{i j} \rho$.

In the transformed space, the governing equation in the local Cartesian system $\mathbf{e}^{\prime}$ becomes

$\frac{\partial p^{\prime}}{\partial x_{i}^{\prime}}=\rho_{i j}^{\prime} \frac{\partial^{2} u_{j}^{\prime}}{\partial t^{2}}, \quad p^{\prime}=\kappa^{\prime} \frac{\partial u_{i}^{\prime}}{\partial x_{i}^{\prime}}$.
Following the same method in Sect. 3, the invariance of governing equation provides the following constraint relation

$\frac{\chi}{\varphi_{i}}=\lambda_{i}^{2}$

The energy conservation provides another two additional constraint conditions

$\alpha \beta_{i}=\frac{\lambda_{i}}{\lambda_{1} \lambda_{2} \lambda_{3}}, \quad \chi_{I} \beta_{i}^{2}=\frac{1}{\lambda_{1} \lambda_{2} \lambda_{3}}$.

As for elastic wave, Eq. (30) has no-unique solution, if we set $\alpha=1$, i.e., $p^{\prime}=p$, then Eq. (30) leads to the following unique solution

$\beta_{i}=\frac{\lambda_{i}}{\lambda_{1} \lambda_{2} \lambda_{3}}, \quad \chi_{i}=\frac{\lambda_{1} \lambda_{2} \lambda_{3}}{\lambda_{i}^{2}}, \quad \varphi_{i}=\lambda_{1} \lambda_{2} \lambda_{3}$.

Finally the transformed relations in a global system are given by

$p^{\prime}=p, \quad \boldsymbol{u}^{\prime}=\frac{\boldsymbol{A} \boldsymbol{u}}{\operatorname{det} \boldsymbol{A}}, \quad \rho^{\prime}=\rho \frac{\operatorname{det} \boldsymbol{A}}{\boldsymbol{A} \boldsymbol{A}^{\mathrm{T}}}, \quad \kappa^{\prime}=\kappa \operatorname{det} \boldsymbol{A}$.

These results agree with those in [18,23], obtained from a completely different method. Since the transformed relation for acoustic wave is not unique, so transformed relation other than Eq. (32) can also be derived.

\subsection{Generalized acoustic wave in pentamode material}

If we consider generalized acoustic wave in the context of pentamode material (PM) [24,34], the corresponding governing equation in the initial space is given by

$\nabla \cdot \sigma=\rho \cdot \ddot{\boldsymbol{u}}, \quad \sigma=\kappa \operatorname{tr}(S \nabla \boldsymbol{u}) \boldsymbol{S}$,

where $\sigma$ is stress tensor and $\sigma=p S, S$ is a general 2-order tensor satisfying $\nabla \cdot S=0, p$ is pseudo-pressure and $\rho$ is mass density tensor. The stiffness tensor of PM is given by $\boldsymbol{C}=\kappa S \otimes S$, which is theoretically feasible [34]. 
Obviously, when $\boldsymbol{\rho}=\rho \mathbf{I}, \boldsymbol{S}=\mathbf{I}$ and $\boldsymbol{\sigma}=p \mathbf{I}$, the classical acoustic wave equation can be recovered. Now we conduct a mapping, which transforms the classical acoustic material in the initial space to a PM in the transformed space, the transformed field and material in the local Cartesian frame $\mathbf{e}^{\prime}$ of the transformed space are related to those in the initial space and scaling factors by

$$
\begin{aligned}
\sigma_{i j}^{\prime} & =\alpha_{I} \delta_{i j} p, \quad u_{i}^{\prime}=\beta_{I} u_{i}, \quad \rho_{i j}^{\prime}=\chi_{I} \delta_{i j} \rho, \\
\kappa^{\prime} & =\varphi \kappa, \quad S_{i}^{\prime}=\eta_{I} \delta_{i j} .
\end{aligned}
$$

Due to form-invariance of the governing equation, we have in the frame $\mathbf{e}^{\prime}$ of the transformed space

$$
\frac{\partial \sigma_{i j}^{\prime}}{\partial x_{i}^{\prime}}=\rho_{i j}^{\prime} \frac{\partial^{2} u_{i}^{\prime}}{\partial t^{2}}, \quad \sigma_{i j}^{\prime}=\kappa^{\prime} S_{K}^{\prime} \frac{\partial u_{k}^{\prime}}{\partial x_{k}^{\prime}} S_{I}^{\prime} \delta_{i j} .
$$

Following the method explained for elastic wave, we have the following constraint relations

$$
\frac{\eta_{1} \beta_{1}}{\lambda_{1}}=\frac{\eta_{2} \beta_{2}}{\lambda_{2}}=\frac{\eta_{3} \beta_{3}}{\lambda_{3}}, \quad \frac{\varphi \eta_{I}^{2}}{\chi_{i}}=\lambda_{i}^{2} .
$$

The conservations for strain potential energy and kinetic energy lead to

$$
\alpha_{I} \beta_{i}=\frac{\lambda_{i}}{\lambda_{1} \lambda_{2} \lambda_{3}}, \quad \chi_{I} \beta_{i}^{2}=\frac{1}{\lambda_{1} \lambda_{2} \lambda_{3}} .
$$

When a mapping is given, $\lambda_{i}$ is known. From Eq. (36), we have non-unique solutions for the scaling factors $\alpha_{i}, \beta_{i}, \chi_{i}, \eta_{i}$ and $\varphi$, we have a freedom to choose the transformed relations differently. For example, if the displacement is kept un-stretched during the mapping, i.e. $u_{i}^{\prime}=u_{i}$ or $\beta_{i}=1$, and set $\eta_{i}=\lambda_{i} /\left(\lambda_{1} \lambda_{2} \lambda_{3}\right)$ as proposed in Ref. [24], unique solution for the other scaling factors are derived as

$\alpha_{i}=\frac{\lambda_{i}}{\lambda_{1} \lambda_{2} \lambda_{3}}, \quad \chi_{i}=\frac{1}{\lambda_{1} \lambda_{2} \lambda_{3}}, \quad \varphi=\lambda_{1} \lambda_{2} \lambda_{3}$.

The corresponding transformations in the global system are given by

$$
\begin{aligned}
\boldsymbol{\sigma}^{\prime} & =\frac{\boldsymbol{A} p}{\operatorname{det} \boldsymbol{A}}, \quad \boldsymbol{u}^{\prime}=\boldsymbol{R} \boldsymbol{u}, \\
\rho^{\prime} & =\frac{\rho}{\operatorname{det} \boldsymbol{A}}, \quad \kappa^{\prime}=\kappa \operatorname{det} \boldsymbol{A} .
\end{aligned}
$$

This result agrees with that proposed by Norris [24] from totally different way, which he used to prevent the mass singularity for acoustic cloaks. Other transformation can also be proposed, more details can be found in Ref. [25].

\subsection{Numerical example for illusion acoustic}

In this section, an acoustic device will be designed, which gives an acoustic illusion: a rigid wall with an entrance will acoustically look like a solid rigid wall. The electromagnetic counterpart has been proposed by Lai et al. [13]. To this end, we choose a square region with rigid boundary and a circular region around the square, both are inside the entrance space between the walls. We perform the following mapping: the rest space of the entrance between the walls is folded into a ring-shaped region (the region between the circle and the square), as shown in Fig. 4a. This action cancels the rest space of the entrance and ring-shaped region, and bonds the square region to the remaining wall. In this case, the mapping $\boldsymbol{A}(\boldsymbol{x})$ can only be evaluated numerically by solving Laplace's equation, proposed by $\mathrm{Hu}$ et al. [35], i.e.,

$\nabla_{x_{i}} x_{i}^{\prime}=0$,

with Dirichlet boundary conditions as mentioned above. Once the transformed tensor is known, the transformed material for such device can be determined by using Eq. (32). To validate the proposed allusion acoustic device, numerical experiment is conducted by COMSOL Multiphysics. The transformed material is firstly evaluated by Laplace's equation mode in COMSOL, then the designed device is illuminated by a point source a distance away from the wall. The background is set to be $\rho=1$ and $\kappa=1$ for illustration purpose. The sound-hard boundary condition $\partial p / \partial n=0$ is used to simulate the wall (rigid boundary), the computed region is surrounded by a PML layer to mimic infinite space. The computed pressure patterns are shown respectively in Fig. 4b, c, d for a rigid wall with an entrance however carefully designed to look acoustically like a solid rigid wall, solid rigid wall and a rigid wall with an entrance. Figure $4 b$ shows clearly that the reflective pressure pattern is the same as a solid rigid wall (Fig. 4c), as expected.

\section{Transformation optics}

5.1 Transformation relation for electromagnetic wave

For EM wave, Maxwell's equations read

$\nabla \times \boldsymbol{E}=-\boldsymbol{\mu} \frac{\partial \boldsymbol{H}}{\partial t}, \quad \nabla \times \boldsymbol{H}=+\boldsymbol{\varepsilon} \frac{\partial \boldsymbol{E}}{\partial t}$.

Due to the symmetry of these equations, we can discuss only the first equation, which is written in a local Cartesian system e of the initial space as

$\ell_{i j k} \frac{\partial E_{k}}{\partial x_{j}}=-\mu_{i j} \frac{\partial H_{j}}{\partial t}$,

where $\ell_{i j k}$ is the Levi-Civita (permutation) symbol. Under a general mapping $\boldsymbol{A}$, the fields $\boldsymbol{E}, \boldsymbol{H}$ and materials $\boldsymbol{\varepsilon}, \boldsymbol{\mu}$ are transported to a physical space, and become $\boldsymbol{E}^{\prime}, \boldsymbol{H}^{\prime}$ and $\boldsymbol{\varepsilon}^{\prime}, \boldsymbol{\mu}^{\prime}$ in the physical space. Using the geometrical constraint conditions proposed in Sect. 2, we have

$$
\begin{aligned}
& \varepsilon_{i j}^{\prime}=\alpha_{I} \alpha_{J} \varepsilon_{i j}, \quad \mu_{i j}^{\prime}=\alpha_{I} \alpha_{J} \mu_{i j}, \\
& E_{i}^{\prime}=\beta_{I} E_{i}, \quad H_{i}^{\prime}=\beta_{I} H_{i},
\end{aligned}
$$


Fig. 4 Simulation of an allusion acoustic device. a The mapping, the pressure pattern for $\mathbf{b}$ rigid wall with the designed entrance, $\mathbf{c}$ a solid rigid wall, $\mathbf{d}$ rigid wall with an entrance a
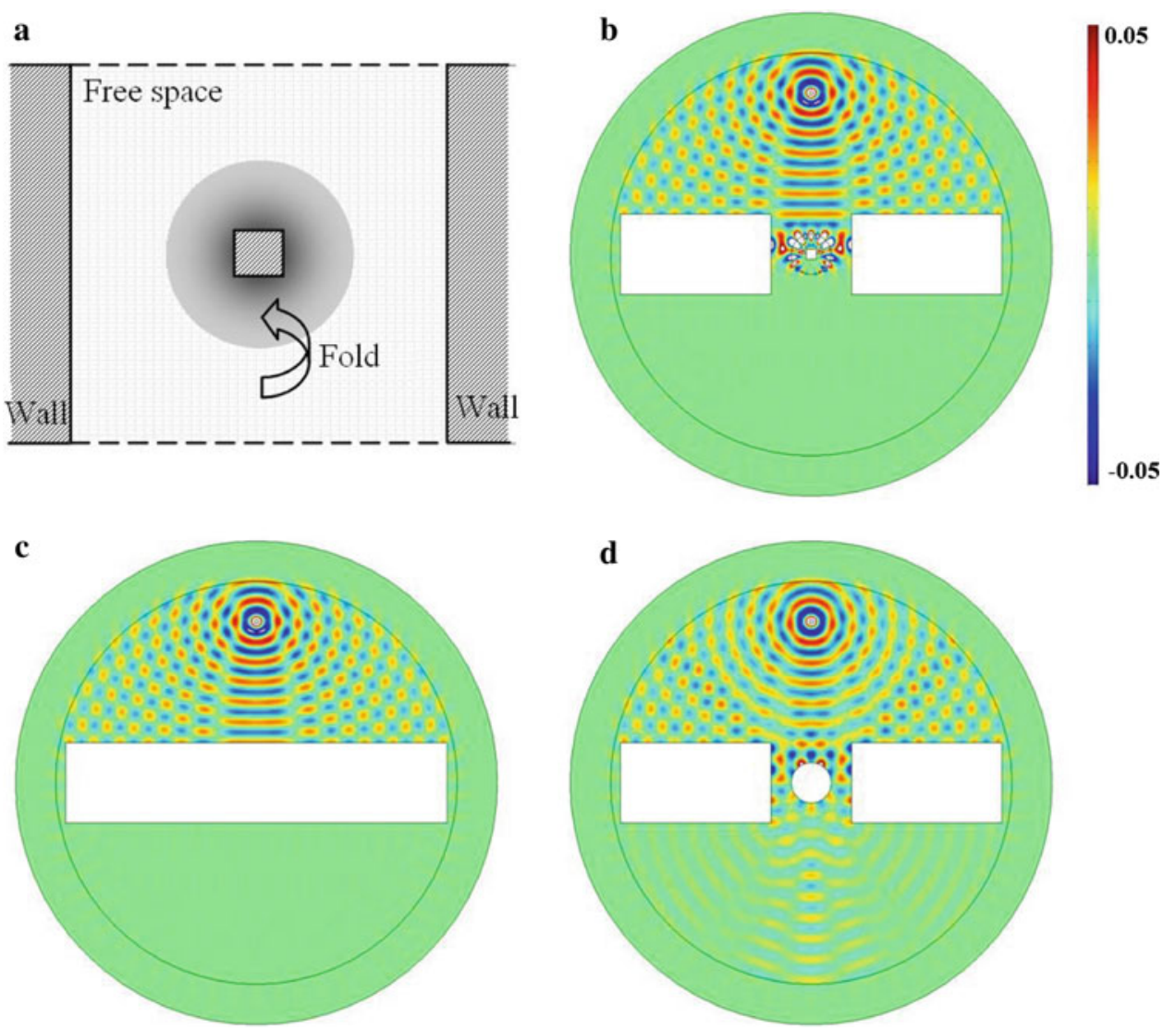

where $\alpha_{i}, \beta_{i}$ are scaling factors for material and field, respectively. In the local Cartesian system $\mathbf{e}^{\prime}$, Eq. (41) can still hold

$\ell_{i j k} \frac{\partial E_{k}^{\prime}}{\partial x_{j}^{\prime}}=-\mu_{i j}^{\prime} \frac{\partial H_{j}^{\prime}}{\partial t}$.

Following the same technique as for elastic wave and acoustic wave, we can express Eq. (43) with the variables in the initial space, scaling factors and stretch tensor, By comparing it with Eq. (41), this provides the following constraint condition

$\frac{\beta_{i}}{\lambda_{j}}=\frac{\beta_{j}}{\lambda_{i}}=\alpha_{k} \alpha_{l} \beta_{l}, \quad$ where $i \neq j, i \neq k, j \neq k, \quad l=1,2,3$.

The energy conservation leads to an additional constraint relation

$\alpha_{i} \alpha_{j} \beta_{I} \beta_{J}=\frac{1}{\lambda_{1} \lambda_{2} \lambda_{3}}$.

Finally the unique relations between the scaling factors and stretch tensor can be derived

$\alpha_{i} \alpha_{j}=\frac{\lambda_{i} \lambda_{j}}{\lambda_{1} \lambda_{2} \lambda_{3}}, \quad \beta_{i}=\frac{1}{\lambda_{i}}$.
So the transformed relations for electromagnetic wave are uniquely determined by

$\varepsilon_{i j}^{\prime}=\frac{\lambda_{i} \lambda_{j}}{\lambda_{1} \lambda_{2} \lambda_{3}} \varepsilon_{i j}, \quad \mu_{i j}^{\prime}=\frac{\lambda_{i} \lambda_{j}}{\lambda_{1} \lambda_{2} \lambda_{3}} \mu_{i j}$,

$E_{i}^{\prime}=\frac{1}{\lambda_{i}} E_{i}, \quad H_{i}^{\prime}=\frac{1}{\lambda_{i}} H_{i}$

Or written in a global system as

$$
\begin{aligned}
\boldsymbol{\varepsilon}^{\prime} & =\frac{\boldsymbol{A} \boldsymbol{\varepsilon} \boldsymbol{A}^{\mathrm{T}}}{\operatorname{det} \boldsymbol{A}}, \quad \boldsymbol{\mu}^{\prime}=\frac{\boldsymbol{A} \boldsymbol{\mu} \boldsymbol{A}^{\mathrm{T}}}{\operatorname{det} \boldsymbol{A}}, \\
\boldsymbol{E}^{\prime}=\left(\boldsymbol{A}^{\mathrm{T}}\right)^{-1} \boldsymbol{E}, & \boldsymbol{H}^{\prime}=\left(\boldsymbol{A}^{\mathrm{T}}\right)^{-1} \boldsymbol{H},
\end{aligned}
$$

which is identical to those given by Pendry et al. [9] from a different method. The transformed relations are unique for EM wave, different from acoustic and elastic waves. In addition, the impedance of the fixed boundary between initial space and physical space for EM wave is guaranteed [36]. There are many interesting devices been designed for EM wave, here we will present an example to design a coating which can make a dielectric circle to look apparently like a rectangle.

\subsection{Numerical example for illusion optics}

Here we will design a device which can make a circle appear as a rectangle. To do this, a virtual space of vacuum with a 

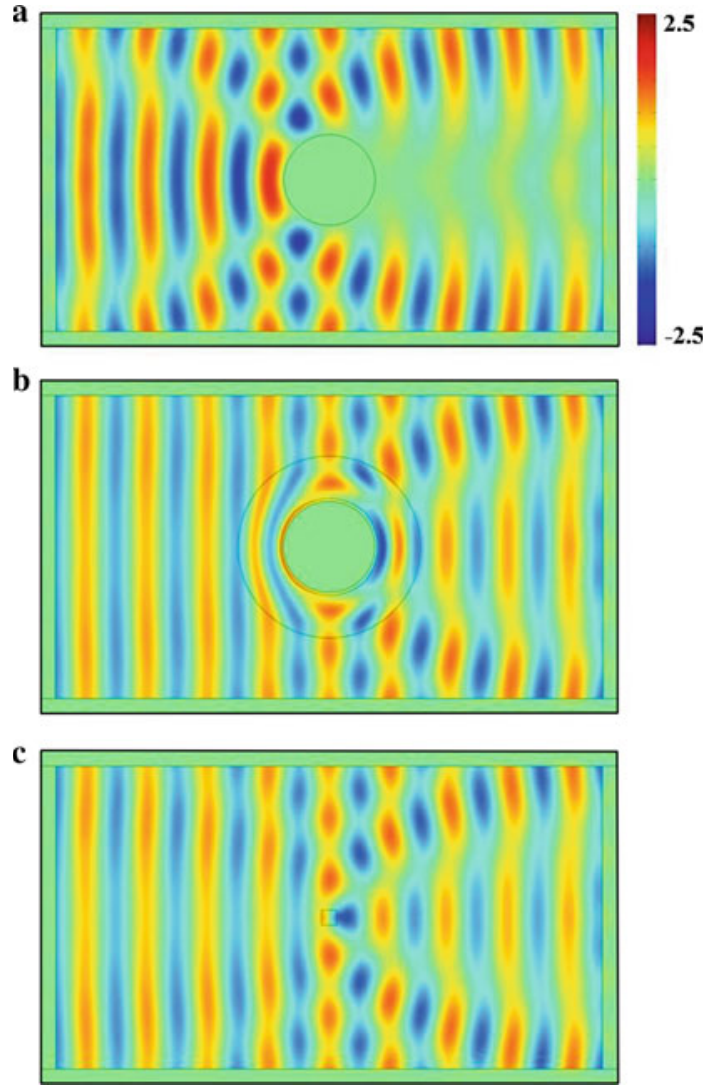

Fig. 5 Simulation of EM illusion cloak: the electric field pattern for plane wave of $0.75 \mathrm{GHz}$ impinging. a A dielectric cylinder, b the designed device, $\mathbf{c}$ adielectric rectangle

rectangle of another material $(\varepsilon=4, \mu=1.5)$ is chosen, we fix a large circle around the rectangle, from the center point of the rectangle, we enlarge to form an another small circle, the rectangle region is also deformed by this mapping. With help of the method proposed by Hu et al. [35], we can evaluate the transformed tensor $\boldsymbol{A}\left(\boldsymbol{x}^{\prime}\right)$ at any point of the deformed region, so as to the corresponding material parameters with Eq. (47). The small circle forms a perfect conductive layer due to the infinite stretch induced by the mapping, so in the physical space, we have a conductive circle coated with a heterogeneous layer, the scattering characteristic of this coated circle is the same as that induced by the dielectric rectangle. Alternatively, we design a coated layer which makes a conductive circle look like a dielectric rectangle.

To validate the design, numerical simulation is performed with the RF mode of COMSOL Multiphysics. A conductive circle, the design device and a dielectric rectangle are illuminated, respectively, by a TE wave of $7.5 \mathrm{GHz}$, the computed regions are surrounded by PML layers. The electric field patterns are shown separately in Fig. 5a-c. From Fig. 5b and $\mathrm{c}$, we find that the scattered electric field of the designed device is almost the same as that by the dielectric rectangle, as expected.

\section{Conclusions}

By interpreting a general mapping as a deformation field, we have figured out the general constraint conditions when a physical process is mapped from an initial space to a physical space. These consist of two geometrical constraints and two physical constraints: the geometrical constraints come from the local conformal mapping round each point, and the physical constraints are obtained by form-invariance of governing equation written in a local Cartesian system and by energy conservation. With help of this general idea, the point by point transformed relations for the field and material are derived in a systematic way for elastic, acoustic and electromagnetic waves. The derived transformed relations are subsequently validated by numerical simulations through designing functional devices. The proposed method can also be applied for other physical processes, e.g., heat transfer and matter waves.

Open Access This article is distributed under the terms of the Creative Commons Attribution Noncommercial License which permits any noncommercial use, distribution, and reproduction in any medium, provided the original author(s) and source are credited.

\section{References}

1. Smith, D.R., Pendry, J.B., Wiltshire, M.C.K.: Metamaterials and negative refractive index. Science 305, 788 (2004)

2. Cai, X.B., Zhu, R., Hu, G.K.: Experimental study for metamaterials based on dielectric resonators and wire frame. Metamaterials 2, 220-226 (2008)

3. Liu, Z.Y., Zhang, X.X., Mao, Y., et al.: Locally resonant sonic materials. Science 289, 1734 (2000)

4. Fang, N., Xi, D., Xu, J. et al.: Ultrasonic metamaterials with negative modulus. Nat. Mater. 5, 452-456 (2006)

5. Lee, S.H., Park, C.M., Seo, Y.M., et al.: Acoustic metamaterial with negative density. Phys. Lett. A 373, 4464-4469 (2009)

6. Yao, S.S., Zhou, X.M., Hu, G.K.: Experimental study on negative effective mass in a $1 \mathrm{D}$ mass-spring system. New J. Phys. 10, 043020 (2008)

7. Bui, H.D.: Fracture Mechanics: Inverse Problems and Solutions. Springer, Dordrecht (2006)

8. Greenleaf, A., Lassas, M., Uhlmann, G.: On nonuniqueness for Calderon's inverse problem. Math. Res. Lett. 10, 685-693 (2003)

9. Pendry, J.B., Schurig, D., Smith, D.R.: Controlling electromagnetic fields. Science 312, 1780 (2006)

10. Leonhardt, U.: Optical conformal mapping. Science 312, 1777 (2006)

11. Schurig, D., Pendry, J.B., Smith, D.R.: Calculation of material properties and ray tracing in transformation media. Opt. Express 14, 9794-9804 (2006)

12. Schurig, D., Mock, J.J., Justice, B.J., et al.: Metamaterial electromagnetic cloak at microwave frequencies. Science 314, 977 (2006)

13. Lai, Y., Chen, H.Y., Zhang, Z.Q., et al.: Complementary media invisibility cloak that cloaks objects at a distance outside the cloaking shell. Phys. Rev. Lett. 102, 093901 (2009)

14. Lai, Y., Ng, J., Chen, H., et al.: Illusion optics: the optical transformation of an object into another object. Phys. Rev. Lett. 102(25), 253902 (2009)

15. Leonhardt, U., Philbin, T.G.: Transformation optics and the geometry of light. Prog. Opt. 53, 69-152 (2009) 
16. Genov, D.A., Zhang, S., Zhang, X.: Mimicking celestial mechanics in metamaterials. Nat. Phys. 5(9), 687-692 (2009)

17. Greenleaf, A., Kurylev, Y., Lassas, M., et al.: Full-wave invisibility of active devices at all frequencies. Comm. Math. Phys. 275(3), 749 (2007)

18. Chen, H., Chan, C.T.: Acoustic cloaking in three dimensions using acoustic metamaterials. Appl. Phys. Lett. 91, 183518 (2007)

19. Zhang, X., Liu, Z.: Negative refraction of acoustic waves in twodimensional phononic crystals. Appl. Phys. Lett. 85(2), 341 (2004)

20. Chen T.Weng, C.N., Chen, J.S.: Cloak for curvilinearly anisotropic media in conduction. Appl. Phys. Lett. 93, 114103 (2008)

21. Zhang, S., Genov, D.A., Sun, C., et al.: Cloaking of matter waves. Phys. Rev. Lett. 100, 123002 (2008)

22. Milton, G.W., Briane, M., Willis, J.R.: On cloaking for elasticity and physical equations with a transformation invariant form. New J. Phys. 8, 248 (2006)

23. Cummer, S.A., Rahm, M., Schurig, D.: Material parameters and vector scaling in transformation acoustics. New J. Phys. 10, 115025 (2008)

24. Norris, A.: Acoustic cloaking theory. Proc. R. Soc. A 464, 2411 (2008)

25. Hu, J., Liu, X.N., Hu, G.K.: Constraint condition on transformed relation for generalized-acoustics and optics (arXiv:0912.5462)

26. Zhou, X.M., Hu, G.K., Lu, T.J.: Elastic wave transparency of a solid sphere coated with metamaterials. Phys. Rev. B. 77, 024101 (2008)
27. Farhat, M., Guenneau, S., Enoch, S.: Ultrabroadband elastic cloaking in thin plates. Phys. Rev. Lett. 103, 024301 (2009)

28. Hu, J., Chang, Z., Hu, G.K.: Controlling elastic waves by transformation media (arXiv.1008.1641)

29. Lai, W.M., Rubin, D., Krempl, E.: Introduction to Continuum Mechanics. 3rd edn. Butterworth-Heinemann, Burlington (1995)

30. Hu, J.: Coordinate transformations method based on deformation view and its application to invisibility design. $\mathrm{PhD}$ Thesis, Beijing Institute of Technology, Beijing (2009) (in Chinese)

31. Milton, G.W., Willis, J.R.: On modifications of Newton's second law and linear continuum elastodynamics. Proc. R. Soc. A 463, 855-880 (2007)

32. Brun, M., Guenneau, S., Movchan, A.B.: Achieving control of in-plane elastic waves. Appl. Phys. Lett. 94, 061903 (2009)

33. Zheng, Y., Huang, X.: Earth Resources Laboratory 2002 Industry Consortium Meeting 1-18 (2002)

34. Milton, G.W., Cherkaev, A.V.: Which elasticity tensors are realizable? J. Eng. Mater. Technol. 117, 483-493 (1995)

35. Hu, J., Zhou, X.M., Hu, G.K.: Design method for electromagnetic cloak with arbitrary shapes based on Laplace's equation. Opt. Express 17, 1308 (2009)

36. Yan, W., Yan, M., Ruan, Z., et al.: Coordinate transformations make perfect invisibility cloaks with arbitrary shape. New J. Phys. 10, 043040 (2006) 